\title{
New Approach to Resonance Crossing
}

\author{
G. Franchetti ${ }^{1}$ and F. Zimmermann ${ }^{2}$ \\ ${ }^{1}$ GSI Darmstadt, Planckstrasse 1, 64291 Darmstadt, Germany \\ ${ }^{2}$ CERN CH-1211, Geneva 23, Switzerland \\ (Received 10 January 2012; published 4 December 2012)
}

\begin{abstract}
Time-varying nonlinear oscillatory systems produce phenomena of resonance crossing and trapping of particles in resonance islands. Traditionally, such processes have been analyzed in terms of adiabatic conditions. Considering, as an example, a simplified one-dimensional model describing the "electroncloud pinch" during a bunch passage in a particle accelerator, here we present an approach to resonance trapping which does not require any adiabatic condition. Instead we introduce the concept of the attraction point and investigate invariance and scaling properties of motion close to the attraction point, considering a single resonance crossing.
\end{abstract}

DOI: 10.1103/PhysRevLett.109.234102

PACS numbers: 41.75.-i, 05.45.-a, 29.27.Bd

Nonlinear oscillatory systems subject to time-varying parameters give rise to the phenomenon of resonance crossing. Trapping or detrapping of a moving object into a resonance occurs during the separatrix crossing process $[1,2]$. This mechanism is found in almost all areas of physics, for example, in tokamaks [3], in planetary science [4-6], in the orbital evolution of asteroid fragments [7], in fluid dynamics [8], in spacecraft research [9], in ion traps [10], and also in accelerator beam physics [11-16].

We here consider resonance-crossing phenomena occurring in an accelerator for a proton beam afflicted by an electron cloud [13,17], which are of relevance, e.g., for the facility for antiproton and ion research in Europe project (FAIR) [18] and for the Large Hadron Collider [19]. During the passage of a proton bunch, the cloud electrons perform nonlinear oscillations in the nonlinear beam potential, which induces a particular "pinch" structure of the negatively charged electron cloud. The electric field from this charge distribution feeds back onto the beam, creating structure resonances as well as phenomena of periodic resonance crossing [13,17,20,21].

The one-turn map describing a specific proton dynamics due to a localized one-dimensional electron layer in a circular accelerator $[17,20,21]$ can be written as

$$
\left(\begin{array}{l}
y_{1} \\
p_{1}
\end{array}\right)=\left(\begin{array}{cc}
\cos \omega & \sin \omega \\
-\sin \omega & \cos \omega
\end{array}\right)\left(\begin{array}{c}
y_{0} \\
p_{0}+\operatorname{sgn}\left(y_{0}\right) \hat{F}
\end{array}\right) .
$$

This equation refers to protons at a certain longitudinal position along the bunch such that they experience the force from a thin electron-cloud layer located in the vertical midplane (at $y=0$ ). The phase-space variables $y$ and $p$ in (1) are the so-called normalized Courant-Snyder coordinates [22], $\omega=2 \pi Q_{y}$ is the betatron phase advance per turn $\left(Q_{y}\right.$ denotes the betatron tune), and $\hat{F}$ represents the effect of the electric field generated by the sheet of electrons located at $y=0$. The "sgn" function incorporates the discontinuity of the Coulomb electric field across the electron sheet layer, approximated as infinitely thin and delta-function-like.

The map (1) creates a series of structure resonances $\mathcal{N} Q_{y}=\mathcal{M}$, with $\mathcal{N}$ and $\mathcal{M}$ integers. We denote with $\delta=\omega-2 \pi \mathcal{M} / \mathcal{N}$ the distance, in betatron frequency, of $Q_{y}$ from the resonance located at $Q_{r}=\mathcal{M} / \mathcal{N}$. By representing the particle coordinates in the complex variable $\mathrm{Y}_{0}=y+i p$, the map (1) takes the convenient form $\mathrm{Y}_{0,1}=$ $e^{-i \omega}\left(\mathrm{Y}_{0,0}+i s_{0} \hat{F}\right)$, where $s_{0}=\operatorname{sgn}\left(y_{0}\right)$. The first index in $\mathrm{Y}$ refers to the reference frame ( 0 for the laboratory reference frame), while the second index refers to the number of iterations of the map. Iterating the map $\mathcal{N}$ times

$$
\mathrm{Y}_{0, \mathcal{N}}=e^{-i \omega \mathcal{N}} Y_{0,0}+i \hat{F} e^{-i \omega \mathcal{N}} \sum_{j=0}^{\mathcal{N}-1} s_{j} e^{i j \omega},
$$

with $s_{j}=\operatorname{sgn}\left[\operatorname{Re}\left(\mathrm{Y}_{0, j}\right)\right]$, allows us to derive an analytic expression for the fixed points, here called $\mathrm{A}_{0}=A_{0, y}+$ $i A_{0, p}$, as

$$
\mathrm{A}_{0}=\frac{\hat{F} e^{-i \delta \mathcal{N} / 2}}{2 \sin (\delta \mathcal{N} / 2)} \sum_{j=0}^{\mathcal{N}-1} s_{j} e^{i j \omega} .
$$

All the fixed points of the same resonance are obtained by rotating the sequence $\left\{s_{j}: j=0, \ldots, \mathcal{N}-1\right\}$ of one fixed point. For the particular case of $\mathcal{M}=1$, the expression for the fixed point closest to the $y$ axis becomes very simple, as there are $\mathcal{N} / 2$ contiguous $s_{j}$ of value +1 and $\mathcal{N} / 2$ contiguous $s_{j}$ equal to -1 leading to

$$
\mathrm{A}_{0}=\frac{\hat{F} \Omega}{2 \tan (\mathcal{N} \delta / 4) \sin (\omega / 2)}
$$

with $\Omega=e^{i(-\pi / 2-\omega / 2)}$. The other fixed points are obtained by repeatedly applying the one-turn map to $A_{0}$.

The dynamics of a particle inside an island is obtained by using the map (2). As the electron-cloud force is discontinuous at $y=0$, there is no island that overlaps with the vertical 
axes. It follows that the second term on the right-hand side of (2) is the same for any particle in the island. Therefore, the $\mathcal{N}$-turn map of a particle in an island with respect to the fixed point $\mathrm{A}_{0}$ is $\mathrm{Y}_{1,1}=e^{-i \omega \mathcal{N}} \mathrm{Y}_{1,0}$; hence, the orbits are circular, and the secondary "island frequency" is $\omega_{s}=\delta$. The first index of $Y, 1$, refers to the reference frame of $A_{0}$. The size of the resonance island [23] of the fixed point (4), i.e., its radius from the $p$ axis, is $S_{0}=\hat{F} /|2 \tan (\mathcal{N} \delta / 4)|$. This discussion shows that the particle dynamics in an island is retrieved by the interpolating Hamiltonian

$$
H_{0}=\frac{\delta}{2}\left|Y_{0}-Z\right|^{2},
$$

where $Y_{0}=(y, p)$ are the coordinates of an arbitrary particle and $Z=\left(Z_{y}, Z_{p}\right)$ designates the coordinates of the fixed point. This Hamiltonian describes the motion of a particle which at instants correspondent to every $N$ th turn coincide with the Poincaré section of the map (1). The procedure of interpolating a map with an interpolating Hamiltonian is guaranteed by the Birkhoff normal form theorem [24], and a general construction technique is presented in Ref. [25]. The applicability of the normal form requires that the fixed point be elliptic.

Suppose now that $\hat{F}$ in (1) is time dependent, $\hat{F}=\hat{F}(N)$, with $N$ denoting the turn number. The dynamical properties of time-dependent systems such as the one described by (1) are often related to the dynamical properties of the "frozen system," in which the time-varying parameter, here $\hat{F}$, is held constant. Because of the change of $\hat{F}$, the "frozen" fixed points created by the structure resonance $Q_{r}=\mathcal{M} / \mathcal{N}$ migrate outward or inward according to Eq. (3) or Eq. (4).

Historically, particles have been considered trapped into a resonance island if the particle coordinates $Y_{0}$ follow the fixed point of the frozen system $\mathrm{A}_{0}(N)$ [11,12]. Namely, when the migration speed of $\mathrm{A}_{0}(N)$ is very slow with respect to the maximum speed of rotation, $v_{r}(N)=$ $S_{0}(N) \delta$, of particles inside the island of the frozen system, an adiabatic regime is reached and particles follow the migration of the island [12]. The ratio of these two velocities, namely, $T=\left|\dot{\mathrm{A}}_{0}(N)\right| / v_{r}(N)$, defines an adiabatic parameter. The adiabatic condition is fulfilled for $T \ll 1$. By contrast, the terminology scattering regime has been used to describe the (nonadiabatic) situation where particles do not follow the frozen fixed points after resonance crossing $[26,27]$. The border between trapping and scattering regimes is not clearly defined, however, and neither is the border between adiabatic and nonadiabatic motion.

In the following, we develop a new characterization of trapping, which does not require the concept of adiabatic motion. We consider a system with a nonlinear one-turn map $Y_{0,1}=M\left(Y_{0,0}\right)$, which describes a dynamics close to a resonance of the order of $\mathcal{N}$. The $\mathcal{N}$ th iteration of the map allows identifying the fixed points $Z=\left(Z_{y}, Z_{p}\right)$ as a solution of $Z=M^{\mathcal{N}}(Z)$. By selecting one of the fixed points $Z$, the dynamics of a particle in the island of $Z$ is described by $Y_{0,1}=M^{\mathcal{N}}\left(Y_{0,0}, Z\right)$. Then by using the Birkhoff normal form an interpolating Hamiltonian $H_{0}=H_{0}\left(Y_{0}, Z\right)$ is found, which allows a description of the dynamics in the island. In the frozen system, $Z$ does not change with time. The motion is described by the canonical equations

$$
\dot{Y}_{0}=\mathbf{S} \nabla H_{0}\left(Y_{0}, Z\right)
$$

with

$$
\mathbf{S}=\left(\begin{array}{cc}
0 & 1 \\
-1 & 0
\end{array}\right)
$$

the symplectic matrix and $\nabla=\left(\partial_{y}, \partial_{p}\right)$. The frozen fixed point $A_{0}$ of the Hamiltonian of the frozen system is found by solving the equation $\nabla H_{0}\left(A_{0}, Z\right)=0$ and keeping $Z$ fixed. The definition of $H_{0}$ implies $A_{0}=Z$.

However, if the fixed point of the frozen system becomes time dependent, i.e., $A_{0}(t)=Z(t)$, the definition and existence of a fixed point are no longer obvious. To investigate this question, we study the motion of a particle in the reference frame of the frozen fixed point $A_{0}(t)$, which is obtained via the coordinate transformation $Y_{1}=Y_{0}-A_{0}$, where $Y_{1}$ are the particle coordinates in the reference frame of $A_{0}$. The dynamics in the reference frame of $A_{0}$ follows from a canonical transformation between the old Hamiltonian $H_{0}\left(Y_{0}, Z\right)$ and the new Hamiltonian $H_{1}\left(Y_{1}, t\right)$ : $H_{1}\left(Y_{1}, t\right)=H_{0}\left(Y_{1}+A_{0}, Z\right)+Y_{1}^{T} \mathbf{S} \dot{A}_{0}$. The structure of this Hamiltonian is now affected by the time-varying parameters via the term $Y_{1}^{T} \mathbf{S} \dot{A}_{0}$. Therefore we can compute again the position of the fixed point, now called $A_{1}$, for the dynamics in the reference frame of $A_{0}$. The frozen fixed point is found by solving $\nabla H_{1}\left(A_{1}, t\right)=0$ and holding $t$ constant, which yields $\nabla H_{0}\left(A_{1}+A_{0}, t\right)+\mathbf{S} \dot{A}_{0}=0$. This equation shows that only if $\dot{A}_{0}=0$ will the point $A_{1}$ not change with time and $A_{1}=0$, therefore, be a fixed point. In case $A_{0}$ varies with time, we may, however, expect that $A_{1}$ varies more slowly than $A_{0}$. We repeat the previous procedure many times, at each step deriving the fixed point $A_{n-1}$ in the reference frame of $A_{n-2}$ and writing the Hamiltonian of the dynamics in the frame of $A_{n-1}$ as

$$
H_{n}\left(Y_{n}, t\right)=H_{0}\left(Y_{n}+\sum_{j=0}^{n-1} A_{j}, Z\right)+Y_{n}^{T} \mathbf{S} \frac{d}{d t}\left(\sum_{j=0}^{n-1} A_{j}\right) .
$$

Following the previous procedure, we find the frozen fixed point $A_{n}$ in the reference frame centered at $A_{n-1}$ from

$$
\nabla H_{0}\left(A_{n}+\sum_{j=0}^{n-1} A_{j}, Z\right)+\mathbf{S} \frac{d}{d t}\left(\sum_{j=0}^{n-1} A_{j}\right)=0 .
$$

In the case of convergence, $A_{n}$ on average varies less and less over subsequent reference frames, and $A_{n} \rightarrow 0$ for $n \rightarrow \infty$. By defining $A=\sum_{j=0}^{\infty} A_{j}$, with $A_{j}$ given by (7), this limit (if it exists) yields the Hamiltonian

$$
H_{\infty}\left(Y_{\infty}, t\right)=H_{0}\left(Y_{\infty}+A, Z\right)+Y_{\infty}^{T} \mathbf{S} \dot{A},
$$


where the frozen fixed point is $A_{\infty}=Y_{\infty}=0$. In this situation the point $A$ satisfies

$$
\nabla_{0} H_{0}(A, Z)+\mathbf{S} \dot{A}=0
$$

where now in (9) $Z$ is taken to be time dependent.

What is the interpretation of (8) and (9)? (i) Equation (8) is the Hamiltonian for the motion of a particle of coordinates $Y$ in the reference frame centered at $A$. (ii) Equation (9) can be rewritten as

$$
\dot{A}=\mathbf{S} \nabla H_{0}(A, Z) .
$$

Surprisingly, this is the equation of motion of a particle of coordinates $A$ in the laboratory reference frame [see (6)]. (iii) If the coordinates of any particle in the island are rescaled with respect to $A$, the particles will circulate around $A$, which is now, by construction, a fixed point. In fact, if one tries to compute the frozen fixed point in the reference frame centered in $A$, from (8) the result is $Y=0$ at any $t$; i.e., $A$ is a dynamic fixed point. We call this point $A$ the attraction point of the system.

If $A$ exists, it can be derived by directly integrating the differential equation (10), including the time dependence in $Z$, rather than summing the series $\sum_{j=0}^{\infty} A_{j}$. Note that in general (10) admits several solutions, while the series which defines $A$ is constructed from $A_{0}$ and therefore is uniquely defined. The correct solution $A$ is obtained by requiring that $A \rightarrow A_{0}$ for $\dot{A}_{0} \rightarrow 0$. That is, for adiabatic motion of $A_{0}$, the attraction point becomes the frozen fixed point.

For the 1D electron cloud map (1), the dynamics of particles inside the resonance island of the frozen system is described by the Hamiltonian (5), which via (10) yields

$$
\dot{\mathrm{A}}+i \delta \mathrm{A}=i \delta \mathrm{Z}
$$

where $\mathrm{Z}=Z_{y}+i Z_{p}$ and $\mathrm{A}=A_{y}+i A_{p}$. We now consider a general type of periodic migration of the frozen fixed point as the consequence of a periodic resonance crossing with fundamental angular frequency $\omega_{c}$ (e.g., the synchrotron oscillation frequency in the case of the electron-cloud map) of the form

$$
\mathrm{A}_{0}(t)=\mathbf{Z}(t)=\sum_{n=0}^{N_{h}}\left[a_{n} \cos \left(n \omega_{c} t\right)+b_{n} \sin \left(n \omega_{c} t\right)\right]
$$

where $N_{h}$ denotes the maximum number of harmonics. The particular values of the complex coefficients $a_{n}$ and $b_{n}$ depend on the detailed shape of the electron pinch and also on the island to which the fixed point belongs. The general solution of (11) for a periodic resonance crossing as in (12) is found by requiring that $A \rightarrow A_{0}$ for $\omega_{c} \rightarrow 0$, which selects the following particular solution of (11):

$$
\begin{aligned}
\mathrm{A}(t)= & \sum_{n=0}^{N_{h}} \frac{a_{n}+i\left(n \omega_{c} / \delta\right) b_{n}}{1-\left(n \omega_{c} / \delta\right)^{2}} \cos \left(n \omega_{c} t\right) \\
& +\sum_{n=0}^{N_{h}} \frac{b_{n}-i\left(n \omega_{c} / \delta\right) a_{n}}{1-\left(n \omega_{c} / \delta\right)^{2}} \sin \left(n \omega_{c} t\right) .
\end{aligned}
$$

If $\omega_{c} \rightarrow 0$, then (13) converges to (12) as it should for an adiabatic crossing. If instead the crossing is fast, i.e., for large $\omega_{c}$, A will significantly differ from $A_{0}$.

As the attraction point $A$ satisfies the equation of the dynamics (11), the quantity $\left|Y_{0}-A\right|^{2}$ is an invariant. In fact, as $Y_{0}$ satisfies Eq. (11) as $A$, then $Y_{0}-A$ satisfies the homogeneous part of (11) from which the invariance follows. For the electron-cloud map this means that the motion of $Y_{0}-A$ is a rotation.

To investigate the effect of single resonance crossing, we consider in the following $\hat{F}$ varying as $\hat{F}(t)=\hat{F}_{\max }\left[1-\cos \left(\omega_{c} t\right)\right] / 2$ for crossing a fourth-order resonance with $\mathcal{M}=1$. Then from (4) we find

$$
\mathrm{A}_{0}(t)=\hat{F}_{\max } \Omega \frac{\left[1-\cos \left(\omega_{c} t\right)\right]}{4 \tan (\mathcal{N} \delta / 4) \sin (\omega / 2)}
$$

The adiabatic parameter $T$ for this type of resonance crossing becomes $T \simeq \sqrt{2} / \tan \left(\omega_{c} t / 2\right) \omega_{c} / \delta$. For example, taking $\omega_{c} / \delta=0.15$, at the first quarter of the frozen fixed point migration, $t_{4}=\pi /\left(2 \omega_{c}\right)$, we find $T \simeq \sqrt{2} \omega_{c} / \delta \simeq$ 0.21 . Therefore according to the adiabatic theory, for $t<t_{4}$, one would expect no trapping of particles due to the nonadiabatic character of the motion, especially if $\left|\mathrm{A}_{0}\left(t_{4}\right)\right|$ is much larger than the beam size. However, nonadiabatic processes leading to trapping can still occur, as there exists the attraction point

$$
\mathrm{A}(t)=\frac{R_{\max }}{2} \Omega\left[1-\frac{\cos \left(\omega_{c} t\right)-i \frac{\omega_{c}}{\delta} \sin \left(\omega_{c} t\right)}{1-\left(\omega_{c} / \delta\right)^{2}}\right],
$$

where $R_{\max }=\hat{F}_{\max } /[2 \tan (\mathcal{N} \delta / 4) \sin (\omega / 2)]$. We observe that the path traced by $\mathrm{A}$ in phase space depends only on $R_{\max }$ and on $\omega_{c} / \delta$ and that it exists for $\left|\omega_{c} / \delta\right|<1$. For $\omega_{c} / \delta=0.15$, at the beginning of the crossing process the attraction point lies outside of the island of the frozen system as illustrated in Fig. 1, top left at the turn $N=35$. Shown in black is the island of the frozen system; the red line represents the path of the frozen fixed point $A_{0}$ and the green line the path of the attraction point $A$. Although the attraction point does not start at the origin, along its later path it may enter the island of the frozen system, when $|\mathrm{A}-\mathrm{Z}|<S_{0}$, as shown in Fig. 1, top right for turn $N=70$. All particles found inside the green circle are trapped and rotate around the moving point $\mathrm{A}$. If the crossing speed is too fast, namely, if $\omega_{c} / \delta>\{2 \sin (\pi / \mathcal{N}) /$ $[1+2 \sin (\pi / \mathcal{N})]\}^{1 / 2}$, the attraction-point path never intercepts the island of the frozen system and trapping is impossible. The opposite happens for slower crossing, as 

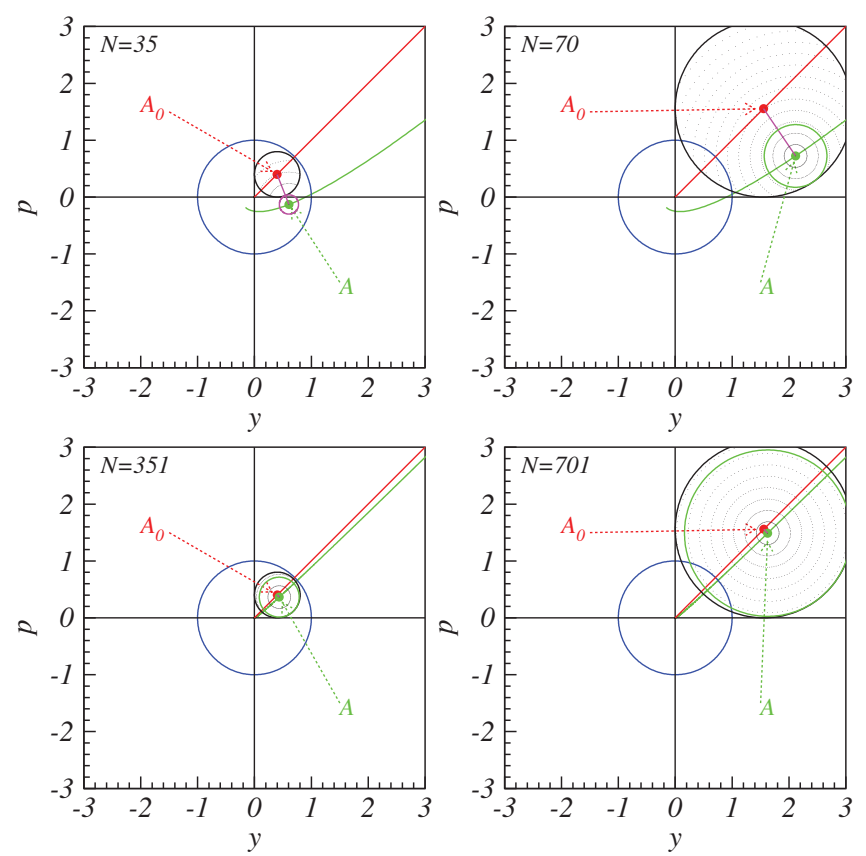

FIG. 1 (color). Position and path of the frozen fixed point (red marker) and of the attraction point (green marker), with $\delta /(2 \pi)=-0.01$, and $\omega_{c} / \delta=0.15$ (top) and $\omega_{c} / \delta=0.015$ (bottom pictures). The blue circle indicates the beam edge assumed in the following simulations.

for $\omega_{c} / \delta=0.015$, where at the corresponding times of Fig. 1 top left and right the attraction point $A$ is found much closer to $A_{0}$ because of the more adiabatic regime (see Fig. 1, bottom left and right).

The dynamical properties of the trapping of particles should be invariant for changes of parameters that leave the path of $A$ invariant. We verify this property by a numerical experiment. We start from a uniform beam distribution with a sharp edge, which we track by repeatedly applying the map (1) with time-varying $\hat{F}(N)$, and after a resonance crossing, i.e., at turn $N=\pi / \omega_{c}$, we count the number of particles inside the island of the attraction point. We choose the maximum migration of the frozen fixed point in the range $2 \leq R_{\max } \leq 100$ in units of the assumed beam size. We also vary the distance from the resonance, considering $\delta /(2 \pi)=-0.1,-0.01$, and -0.001 . By setting $R_{\max }$ and $\delta, \hat{F}_{\max }$ is determined. For each pair of $R_{\max }$ and $\delta$, a curve of the fraction of trapped particles is plotted versus $\omega_{c} / \delta$, with the result shown in Fig. 2. This picture demonstrates the property of invariance of the number of trapped particles. The green, red, and black curves overlap according to $R_{\max }$. Note that at $\omega_{c} / \delta=0.15$ (dashed line in Fig. 2) significant particle trapping is still detected: For example, for the curves $R_{\max }=100$, about $25 \%$ of the particles are trapped and transported to amplitudes equal to 100 times the beam size, although during the passage through the initial beam area $T \geqslant 1$. This is possible because of the existence of the attraction point.

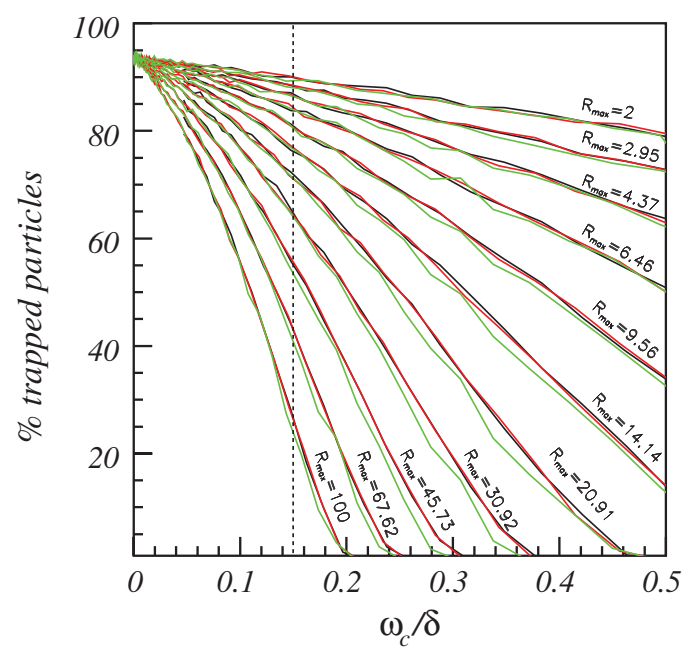

FIG. 2 (color). Simulated trapping efficiency for different distances to the resonance equal to $\delta /(2 \pi)=-0.1,-0.01$, and -0.001 (green, red, and black curves, respectively), demonstrating invariance for trapping.

The observed scaling behavior is noteworthy. Changing $\delta$ and requiring $R_{\max }$ to remain constant automatically varies the strength of the structure resonance, because the maximum $\hat{F}$ is consistently readjusted according to (4). The number of turns needed for crossing the resonance $N_{c}$ is, however, diminished for increasing $\delta$ and constant $R_{\max }$. Our results suggest a strategy for long-term simulations in which the leading structure resonance created by the electron cloud could be artificially enhanced, keeping the pinch process frozen, reducing then the computational time for modeling long-term beam storage. A later scaling of these results could bring the long-term modeling down to a realistic time scale. A similar strategy could be applied for modeling the periodic crossing induced by space charge, e.g., in the FAIR project [28].

In summary, we have presented a new approach to describing resonance-trapping phenomena in time-varying nonlinear oscillatory systems. Specifically, from the trajectory of the frozen fixed point, we have constructed a new point, the attraction point, which determines the particle motion inside the frozen resonance islands and the overall particle trapping efficiency. This approach was applied to the electron-cloud map (1) and the result found to be in excellent agreement with direct numerical simulations. Associated invariance and scaling properties have been highlighted.

Complementary studies confirm that the concept of the attraction point also applies to other nonlinear maps, such as a cubic map, although in this case the procedure to find the attraction point has been purely numerical.

[1] A. I. Neishtadt, J. Appl. Math. Mech. 39, 594 (1975).

[2] J. L. Tennyson, J. R. Cary, and D. F. Escande, Phys. Rev. Lett. 56, 2117 (1986). 
[3] R. Kamendje, S. V. Kasilov, W. Kernbichler, I. V. Pavlenko, E. Poli, and M.F. Heyn, Phys. Plasmas 12, 012502 (2005).

[4] C. D. Murray and S. F. Dermott, Solar Systems Dynamics (Cambridge University Press, Cambridge, England, 2000).

[5] A. C. M. Correia and J. Laskar, Nature (London) 429, 848 (2004).

[6] K. Tsiganis, R. Gomes, A. Morbidelli, and H. F. Levison, Nature (London) 435, 459 (2005).

[7] C. Froeschlé and H. Scholl, in Proceedings of the Asteroids Comets Meteors 1991 Conference, Flagstaff, 1991 (unpublished).

[8] T. H. Solomon and I. Mezic, Nature (London) 425, 376 (2003).

[9] M. Yu. Zabolotnov, Cosmic Res. (Transl. of Kosm. Issled.) 41, 171 (2003).

[10] H. Okamoto, in Proceedings of PAC 2011, New York (JACoW, New York, 2011), p. 46.

[11] A. Schoch, CERN Report No. CERN 57-23, 1958.

[12] A. W. Chao and M. Month, Nucl. Instrum. Methods 121, 129 (1974).

[13] E. Benedetto, G. Franchetti, and F. Zimmermann, Phys. Rev. Lett. 97, 034801 (2006).

[14] G. Franchetti and I. Hofmann, Nucl. Instrum. Methods Phys. Res., Sect. A 561, 195 (2006).

[15] K. Ohmi and K. Oide, Phys. Rev. ST Accel. Beams 10, 014401 (2007).
[16] I. Hofmann et al., in Proceedings of the Thirty-ninth ICFA Workshop, (JACoW, Tsukuba, 2006), p. 268; S. Y. Lee, G. Franchetti, I. Hofmann, F. Wang, and L. Yang, New J. Phys. 8, 291 (2006).

[17] G. Franchetti, I. Hofmann, W. Fischer, and F. Zimmermann, Phys. Rev. ST Accel. Beams 12, 124401 (2009).

[18] P. Spiller and G. Franchetti, Nucl. Instrum. Methods Phys. Res., Sect. A 561, 305 (2006).

[19] O. Brüning et al., LHC Design Report 1. The LHC Main Ring. CERN-2004-003, 2004.

[20] G. Franchetti and F. Zimmermann, in Proceedings of IPAC 2010, Kyoto, Japan (unpublished).

[21] G. Franchetti and F. Zimmermann, Report No. CERN2008-005.

[22] E. D. Courant and H. S. Snyder, Ann. Phys. (Paris) 3, 1 (1958).

[23] B. V. Chirikov, Phys. Rep. 52, 263 (1979); A. J. Lichtenberg and M. A. Lieberman, Regular and Chaotic Dynamics (Springer-Verlag, Berlin, 1983).

[24] G. D. Birkhoff, Dynamical Systems (American Mathematical Society, Providence, 1927).

[25] A. Bazzani et al., CERN Report No. 94-02, 1994.

[26] A. I. Neishtadt, Sov. J. Plasma Phys. 12, 568 (1986).

[27] A. I. Neishtadt and A. A. Vasiliev, Nucl. Instrum. Methods Phys. Res., Sect. A 561, 158 (2006).

[28] G. Franchetti et al., Phys. Rev. ST Accel. Beams 13, 114203 (2010). 